\title{
THE EXISTENCE OF TRAVELLING WAVES FOR PHASE FIELD EQUATIONS AND CONVERGENCE TO SHARP INTERFACE MODELS \\ IN THE SINGULAR LIMIT
}

\author{
BY \\ G. CAGINALP (University of Pittsburgh, Pittsburgh, Pennsylvania) \\ AND \\ Y. NISHIURA ( Hiroshima University, Hiroshima, Japan)
}

1. Introduction. In this paper we prove the existence of travelling wave solutions to phase field equations in a scaling regime for which a sharp interface model (encompassing surface tension and kinetic undercooling) is attained as a singular limit. Furthermore, we prove that the distinguished limits which lead to sharp interface models which neglect surface tension or kinetic undercooling do not support travelling wave solutions with the same boundary conditions.

The phase field equations are a parabolic system describing the (dimensionless) temperature, $u(t, x)$, (which is scaled so that $u=0$ is the ordinary planar equilibrium melting or freezing temperature) and an "order parameter" or phase field, $\varphi(t, x)$ at time $t \in \mathbb{R}^{+}$and spatial point $x \in \Omega \subset \mathbb{R}^{d}$. The phase field, $\varphi$, is scaled so that $\varphi$ near +1 is associated with the liquid phase and $\varphi$ near -1 with the solid. The interface is defined implicitly as the set of points for which $\varphi$ vanishes. Further discussion and references can be found in [1], [2]. The basic phase field equations can be written as

$$
\begin{gathered}
\alpha \xi^{2} \varphi_{t}=\xi^{2} \Delta \varphi+a^{-1} g(\varphi)+2 u, \\
u_{t}+\frac{l}{2} \varphi_{t}=K \Delta u,
\end{gathered}
$$

where $l$ and $K$ are positive constants representing (dimensionless) latent heat and diffusivity, respectively. The function $g$ is a derivative of a symmetric double well potential with minima at \pm 1 , e.g., $g(\varphi)=\frac{1}{2}\left(\varphi-\varphi^{3}\right)$.

This double well can be viewed in terms of a probabilistic measure on the individual atoms. The extent to which this measure discriminates against the interfacial region and in favor of the liquid or solid phases depends on how close $a$ is to zero.

The parameters $\alpha$ and $\xi$ also have microscopic definitions as well as macroscopic interpretations. In particular, $\tau=\alpha \xi^{2}$ is a microscopic relaxation time which can be 
related to macroscopic quantities by means of experiments such as light scattering. The parameter $\xi$ is a dimensionless length scale which represents the strength of microscopic interactions, since the $\xi^{2} \Delta \varphi$ term is derived from the original microscopic interaction term $\sum_{x, x^{\prime}} J\left(x-x^{\prime}\right) \varphi(x) \varphi\left(x^{\prime}\right)$. Then Eq. (1.1) arises from a Landau-Ginzberg free energy of the form

$$
\mathscr{F}\{\varphi\}=\int_{\Omega} d x\left\{\frac{\xi^{2}}{2}(\nabla \varphi)^{2}-G(\varphi)-2 u \varphi\right\}
$$

where $G^{\prime}(\varphi)=g(\varphi)$. In equilibrium, one expects $\varphi$ to be a minimizer of $\mathscr{F}$ so that $\delta \mathscr{F} / \delta \varphi=0$. When the material is not in equilibrium, it is assumed to be moving toward equilibrium under the influence of a "force" which is proportional to the extent it is away from equilibrium, i,e., $\tau \varphi_{t}=-\delta \mathscr{F} / \delta \varphi$. For a wide spectrum of phase boundary problems, including ordinary phase transitions for which the interfacial width is not large, $\xi$ and $a$ can be regarded as small parameters.

Equations (1.1), (1.2) can be studied subject to initial conditions

$$
u(0, x)=\tilde{u}(x), \quad \varphi(0, x)=\tilde{\varphi}(x), \quad x \in \Omega
$$

and appropriate boundary conditions such as

$$
u(t, x)=u_{\partial}(x), \quad \varphi(t, x)=\varphi_{ \pm}(x), \quad x \in \partial \Omega
$$

where $\varphi_{ \pm}$are the right and left roots of $a^{-1} g(\varphi)+2 u=0$. Since $a$ is a small parameter, these roots will be approximately \pm 1 . The second condition in (1.4) is natural since it is compatible with $\varphi_{t}=\Delta \varphi=0$ in (1.1), so that $\varphi$ is constant when it is far from the interface.

The macroscopic significance of the parameters is best observed by defining parameters

$$
\epsilon \equiv \xi a^{1 / 2}, \quad \hat{\sigma} \equiv \xi a^{-1 / 2} .
$$

By multiplying (1.1) by $a$, it is evident that $\epsilon$ is the length scale which measures the width of the transition layer for $\varphi$, i.e., the width of the interface between solid and liquid. This is borne out in the rigorous treatments of the stationary transition layers as well.

The parameter $\hat{\sigma}$ is related to the surface tension which can be defined by a local interpretation of

$$
\sigma \equiv \frac{\mathscr{F}(\varphi)-\frac{1}{2} \mathscr{F}\{+1\}-\frac{1}{2} \mathscr{F}\{-1\}}{\text { Interface area }}
$$

so that $\sigma$ is the normalized difference between the free energies with and without an interface.

If we define $\Phi(y)$ as the solution of the problem (see also Lemma 2.1)

$$
\Phi_{y y}+g(\Phi)=0, \quad \Phi( \pm \infty)= \pm 1, \quad \Phi_{y}( \pm \infty)=0,
$$

and let $m \equiv\left\|\Phi_{y}\right\|_{L^{2}(\mathbb{R})}^{2}$, then (1.6) can be calculated to leading order in $\epsilon$ as

$$
\sigma_{0}=\left\|\Phi_{y}\right\|_{L^{2}(\mathbb{R})}^{2} \xi a^{-1 / 2}=m \hat{\sigma}
$$


We note that while $\sigma_{0}$ is independent of $\epsilon$ if $\hat{\sigma}$ is held constant, the exact surface tension, $\sigma$, involves the $L^{2}$ norm of the true solution, $\varphi$, and therefore depends on $\epsilon$. However, for our purposes, we may assume that surface tension is given by $\sigma_{0}$, since $\sigma_{0}-\sigma(\epsilon)=O(\epsilon)$ and $\lim _{\epsilon \rightarrow 0} \sigma(\epsilon)=\sigma_{0}>0$.

In considering various singular limits of $(1.1),(1.2)$ it is very useful to note the behavior of $\epsilon$ and $\sigma_{0}$ in terms of the effects on the resulting macroscopic equations. In particular, it is clear that a sharp interface model requires a limit as $\epsilon$ approaches zero. However, one may have $\xi$ and $a$ in various distinct scalings while $\epsilon \rightarrow$ 0 . If one chooses a scaling in which $\hat{\sigma}$ approaches zero, then the surface tension approaches zero as the interfacial thickness vanishes. However, it is clear from (1.5) that the distinguished limit of finite surface tension ( $\hat{\sigma}$ held fixed as $\epsilon \rightarrow 0$ ) can also be considered.

These distinguished limits of the phase field equations were considered in [1], [2] with the formal asymptotic result that the major sharp interface problems (which are defined below) are each distinct limiting cases of (1.1), (1.2). For this purpose, it is convenient to rewrite (1.1) by using (1.5), (1.7) as

$$
\alpha \epsilon^{2} \varphi_{t}=\epsilon^{2} \Delta \varphi+g(\varphi)+\frac{2 \epsilon m}{\sigma_{0}} u
$$

We now define the relevant sharp interface models which assume that the two phases, e.g., solid and liquid, are separated by a sharp interface, $\Gamma(t)$. The heat diffusion equation applies in each phase and the latent heat of fusion across the interface must be dissipated into the two phases, leading to the equations,

$$
\begin{gathered}
u_{t}=K \Delta u, \quad \text { on } \Omega \backslash \Gamma, \\
l v=K[\nabla u \cdot n]_{+}^{-}, \quad \text { on } \Gamma,
\end{gathered}
$$

where $l$ and $K$ are defined as before, $v$ is normal velocity of the interface, and []$_{+}^{-}$denotes the jump in the normal derivative of $u$ from solid to liquid. The first mathematical model of solidification, known as the classical Stefan model [3], stipulates the temperature condition

$$
u=0 \quad \text { on } \Gamma
$$

as the additional interface condition.

In this classical problem, the temperature serves a double role in that the sign of $u$ determines phase. Once the physical phenomena of supercooling (and analogously superheating), or the presence of liquid at subzero temperatures is introduced, it is clear that this dual role is no longer possible. Nevertheless, replacing (1.11) with a physically more accurate equation for the interface results in a system of equations which has been studied recently in the physics and applied mathematics literature. In particular, a modified Stefan problem is obtained by coupling (1.9), (1.10) with

$$
u=-\frac{\sigma}{\Delta s} \kappa-\alpha \frac{\sigma}{\Delta s} v
$$

where $\kappa$ is the sum of principal curvatures and $\Delta s$ is the entropy difference between 
the two phases (assumed to be constant). An alternative modified Stefan problem is obtained by neglecting the $-\alpha \sigma v / \Delta s$ term in (1.12).

Equations (1.9), (1.10), (1.11) or (1.12) are subject to initial and boundary conditions such as the $u$ part of (1.3), (1.4).

It was shown in [1], [2] that the formal asymptotic limits of the phase field equations depending on each scaling regime are given as follows: (1.2), (1.8) approach the classical Stefan model (resp. modified Stefan model) in the limit of $\epsilon, \hat{\sigma} \rightarrow 0$ (resp. $\epsilon \rightarrow 0, \hat{\sigma}$ held fixed) for a positive $\alpha$. If $\alpha$ also tends to zero as well as $\epsilon \rightarrow 0, \hat{\sigma}$ held fixed, we obtain an alternative modified Stefan model.

It is well known that the surface tension $\sigma_{0}$ is of crucial importance as a stabilizing influence in the shape evolution of the macroscopic interface. Clearly, from (1.12), a large surface tension tends to inhibit the development of a large curvature as the interface evolves in time. The role of the kinetic undercooling term $-\alpha \sigma v / \Delta s$ in (1.12) has also been of interest more recently [8], [9]. This term does not alter the stability-instability spectrum but tends to decrease the magnitude of the instability for the unstable modes. Another important feature of this term is that under appropriate conditions, travelling wave solutions are possible for (1.9)-(1.12) if and only if $\alpha \neq$ 0 .

The relationship between the phase field equations (1.2), (1.8) and these sharp interface problems is of both theoretical and practical interest. In addition to proving the possibility of numerical approximation of sharp interface problems, the phase field equations offer a new avenue for proving theorems on such sharp interface problems. In particular, methods such as those of dynamical systems are applicable to parabolic equations including the phase field model, but not directly to Stefan-type problems since the latter involve interface conditions with discontinuous gradients.

The convergence of a system of parabolic equations such as (1.2), (1.8) in the singular limit as $\epsilon \rightarrow 0$ is a delicate problem. In a scaling which formally approaches the classical Stefan model, (1.9)-(1.11), one must also have $\sigma_{0} \rightarrow 0$ in (1.8), thereby creating an additional singular limit. Some of the theoretical difficulties and the notorious instabilities of the interface in the classical Stefan model are clear from this perspective.

An important set of problems is the rigorous justification that solutions to the phase field equations within various scaling regimes have asymptotic limits that are governed by solutions to sharp interface, Stefan-type problems. Such rigorous theorems have been limited thus far to steady state problems [4]-[7]. In particular, it has been proven that solutions to the steady state phase field equations $\left(u_{t}=\varphi_{t}=0\right)$ converge to those of the steady state modified Stefan problem. The latter simply involves finding a function $u$ and a curve (or surface) $\Gamma$ such that at any point $x \in \Gamma, u(x)$ is proportional to the mean curvature at $x$. Part of the difficulty in proving analogous theorems for the dynamical situations is the scarcity of results for problems such as the time-dependent, modified Stefan problem.

In this paper we present the first rigorous convergence results in the dynamical setting. Namely, we prove the existence of travelling waves for the phase field equations (1.2), (1.3) for small $\epsilon$ with $\sigma_{0}$ held fixed, and prove convergence to the modified Stefan model (1.9), (1.10), (1.12). 
For the modified Stefan problem, one can verify the existence [8], [9] of the following travelling wave solution with velocity $c^{*}$ and $u(t,+\infty)=u_{\text {cool }}$ :

$$
\begin{aligned}
u(t, x) & = \begin{cases}u_{\mathrm{cool}}+l e^{-\left(c^{*} / K\right)\left(x-c^{*} t\right)}, & x>c^{*} t, \\
u_{\mathrm{cool}}+l, & x \leq c^{*} t,\end{cases} \\
c^{*} & \equiv \frac{-4}{\alpha \sigma_{0}}\left(u_{\mathrm{cool}}+l\right) .
\end{aligned}
$$

The boundary condition, $u_{\text {cool }}$, must satisfy the same constraint imposed on the phase field solutions (see (2.11)).

The physical problem here is that the material is in a liquid state for $x>c^{*} t$ and solid for $x<c^{*} t$. By the constraint imposed on the boundary condition at $+\infty$, the temperature is always negative, and the freezing continues as a result of the lower temperature in the liquid. Thus the boundary condition $u(t,+\infty)=u_{\text {cool }}$ is the driving force which maintains the constant velocity planar wave.

For travelling wave solutions to the phase field equations (see (2.2) and (2.3)), we define the boundary conditions, with $z \equiv x-c t$, as

$$
\begin{aligned}
& u(-\infty) \equiv u_{s}^{\epsilon}, \quad \varphi(-\infty)=\varphi_{-\infty}^{\epsilon}, \\
& u(\infty)=u_{\text {cool }}<0, \quad \varphi(\infty)=\varphi_{\infty}^{\epsilon}, \\
& \varphi_{z}( \pm \infty)=0=u_{z}( \pm \infty)
\end{aligned}
$$

where $\varphi_{-\infty}^{\epsilon}$ is the left-most root of $g(\varphi)+\left(2 \epsilon m / \sigma_{0}\right) u_{s}^{\epsilon}=0$, and $\varphi_{\infty}^{\epsilon}$ the right-most root of $g(\varphi)+\left(2 \epsilon m / \sigma_{0}\right) u_{\text {cool }}=0$. Note that the above boundary conditions are not independent; in fact, we will see in Sec. 2 that once $u_{\text {cool }}$ is given, other data are uniquely determined as functions of $\epsilon$.

Thus, the problem is to prove the existence of steady planar solutions to (1.2), (1.8), (1.14) for small $\epsilon$, and to show that the temperature $u$ converges in an appropriate norm to the solutions (1.13) as $\epsilon$ approaches zero. To do this, we will employ the alternative method to reduce the entire problem to solving the bifurcation equation $B(c, \epsilon)=0$ (see (2.32)).

Mathematically it is not a priori clear how to choose the solution space which is valid uniformly up to $\epsilon=0$ since, when $\epsilon \downarrow 0$, the phase function $\varphi$ approaches a discontinuous function in the original coordinate $z$. Even if we take a stretched coordinate $y=\frac{z}{\epsilon}$, then temperature, $u$, does not have a limit in $\mathscr{B}(\mathbb{R})$ which satisfies the boundary conditions (see Remark 2.3). However we can show that the scaled bifurcation equation $\widetilde{B}(c, \epsilon)=0$ is valid up to $\epsilon=0$ (see Lemma 2.5) and the limiting velocity $c^{*}$ is uniquely determined as the zero point of $\widetilde{B}(c, 0)=0$.

If $\xi$ and $a$ tend to zero so that $\epsilon$ and $\hat{\sigma}$ defined by (1.5) both approach zero, then the formal limit of the phase field equation is the classical Stefan model, for which the travelling wave solutions of the form (1.13) are not possible under the constraint considered (i.e., (2.11)). In Sect. 3 we prove within this scaling limit that travelling waves cannot exist subject to the same conditions.

Recently, travelling wave solutions to phase field equations in a different scaling regime (i.e., $a=$ constant) have been studied numerically [13]. Also the transi- 
tion from planar travelling fronts to curved ones for phase field equations has been obtained in [14] for a different scaling regime (i.e., $a=$ constant and $\alpha=O\left(\xi^{-1}\right)$ ).

2. Existence of travelling wave solutions and their singular limit. We seek travelling wave solutions to $(1.2),(1.8),(1.14)$ which move at constant velocity $c$ in the positive $x$ direction. With moving coordinate

$$
z \equiv x-c t
$$

Eqs. (1.8), (1.2) become

$$
\begin{gathered}
\epsilon^{2} \varphi_{z z}+\alpha \epsilon^{2} c \varphi_{z}+g(\varphi)+\frac{2 \epsilon m}{\sigma_{0}} u=0, \\
K u_{z z}+c\left(u_{z}+\frac{l}{2} \varphi_{z}\right)=0 .
\end{gathered}
$$

Integrating the second equation (2.3) from $z=-\infty$ we have, upon utilizing the boundary conditions (1.14)

$$
K u_{z}+c\left\{\left(u-u_{s}^{\epsilon}\right)+\frac{l}{2}\left(\varphi-\varphi_{-\infty}^{\epsilon}\right)\right\}=0 .
$$

The associated first-order system for $(2.2),(2.4)$ is given by

$$
\begin{gathered}
\epsilon \varphi_{z}=\chi, \\
\epsilon \chi_{z}=-\alpha \epsilon c \chi-g(\varphi)-2 \frac{\epsilon m}{\sigma_{0}} u, \\
u_{z}=-\frac{c}{K}\left\{\left(u-u_{s}^{\epsilon}\right)+\frac{l}{2}\left(\varphi-\varphi_{-\infty}^{\epsilon}\right)\right\} .
\end{gathered}
$$

The travelling wave for (2.2), (2.3) corresponds to a heteroclinic orbit of (2.5)(2.7) which connects two distinct equilibria. The equilibria of (2.5)-(2.7) are given by the intersection of two curves

$$
g(\varphi)+\frac{2 \epsilon m}{\sigma_{0}} u=0 \quad \text { and } \quad u-u_{s}^{\epsilon}+\frac{l}{2}\left(\varphi-\varphi_{-\infty}^{\epsilon}\right)=0 .
$$

The boundary conditions (1.14) imply that for sufficiently small $\epsilon$ there are always three equilibria $L^{\epsilon}, S^{\epsilon}$, and $I^{\epsilon}$ in the $x=0$-plane as shown in Fig. 1 . Note that the condition $u_{\text {cool }}<0(1.14)$ implies that the location of each equilibrium is uniquely determined as a function of $\epsilon$. We are interested in a heteroclinic orbit connecting $L^{\epsilon}=\left(\varphi_{\infty}^{\epsilon}, 0, u_{\text {cool }}\right)$ to $S^{\epsilon}=\left(\varphi_{-\infty}^{\epsilon}, 0, u_{s}^{\epsilon}\right)$. From (2.8) and (1.14) one clearly has the limits

$$
\lim _{\epsilon \downarrow 0} \varphi_{ \pm \infty}^{\epsilon}= \pm 1, \quad \lim _{\epsilon \downarrow 0} u_{s}^{\epsilon}=u_{\text {cool }}+l .
$$

More precisely, we have

$$
\varphi_{ \pm \infty}^{\epsilon} \mp 1=\epsilon \eta_{ \pm}(\epsilon), \quad u_{s}^{\epsilon}-\left(u_{\text {cool }}+l\right)=\epsilon \rho(\epsilon),
$$

where $\eta_{ \pm}(\epsilon), \rho(\epsilon)$ are smooth and bounded functions up to $\epsilon=0$. 


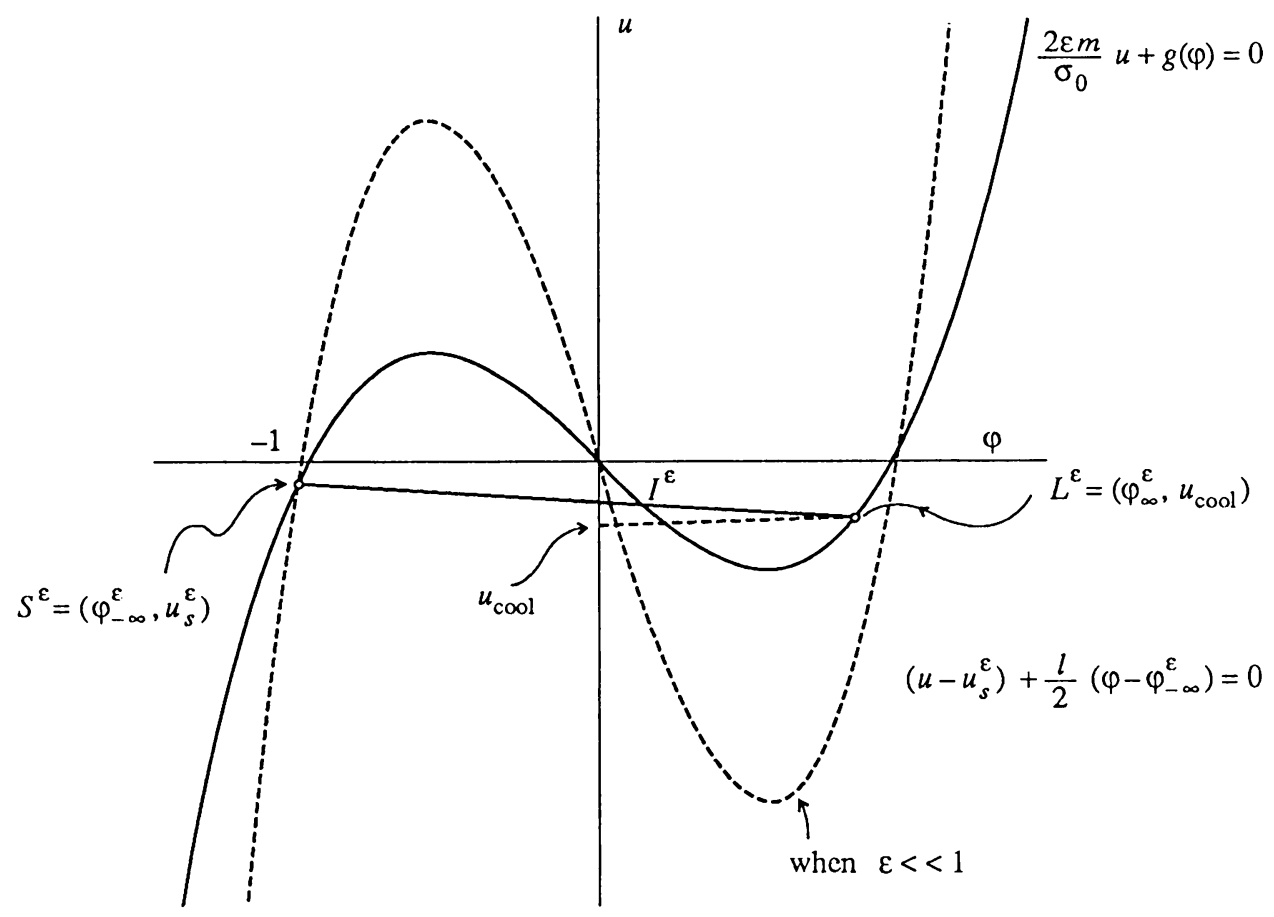

FIG. 1.

It will be seen later that $(2.9 \mathrm{~b})$ gives the temperature at the interface of the planar travelling wave of the modified Stefan model (1.9), (1.10), (1.12). To ensure the positivity of the velocity $c$, we will need to assume

$$
u_{\text {cool }}+l<0 \text {. }
$$

That is, the temperature at infinity must be low in comparison with the latent heat. Physically, this means that the supercooling must overcome the amount of latent heat released at the interface in order to perpetuate the solidification at constant velocity. To avoid the ambiguity of shift invariance, we subsequently fix the phase as

$$
\varphi(0)=0
$$

Our main result is the following.

Theorem 2.1. Under (2.11) with $\hat{\sigma}$ fixed (and positive) there exists $\epsilon_{0}>0$ such that (2.2), (2.3) has a unique $\epsilon$-family of classical solutions $\left(\varphi^{\epsilon}(z), u^{\epsilon}(z)\right)$ for $0<\epsilon \leq \epsilon_{0}$ with velocity $c=c(\epsilon)$ connecting $L^{\epsilon}=\left(\varphi_{\infty}^{\epsilon}, 0, u_{\text {cool }}\right)$ to $S^{\epsilon}=\left(\varphi_{-\infty}^{\epsilon}, 0, u_{s}^{\epsilon}\right)$ such that $c(\epsilon)$ is a continuous function of $\epsilon$ on $\left[0, \epsilon_{0}\right]$ and $\left(\varphi^{\epsilon}, u^{\epsilon}\right)$ has the following 
limiting behavior for any $A>0$ :

$$
\begin{aligned}
& \lim _{\epsilon \downarrow 0} \varphi^{\epsilon}= \begin{cases}-1 & \text { uniformly on } z \in(-\infty, A], \\
1 & \text { uniformly on } z \in(A, \infty),\end{cases} \\
& \lim _{\epsilon \downarrow 0} u^{\epsilon}= \begin{cases}u_{\text {cool }}+l, & \text { for } z \leq 0, \\
u_{\text {cool }}+l e^{-\left(c^{*} / K\right)\left(x-c^{*} t\right)} & \text { for } z>0,\end{cases}
\end{aligned}
$$

where the limit is uniform on $\mathbb{R}$ with respect to the distance measure given by the travelling coordinate $z=x-c(\epsilon) t$. Here $c^{*}$ is the limiting velocity given by

$$
c^{*}=\lim _{\epsilon \downarrow 0} c(\epsilon)=-\frac{4}{\alpha \sigma_{0}}\left(u_{\text {cool }}+l\right) .
$$

That is, $\left(\varphi^{\epsilon}, u^{\epsilon}\right)$ converges to the travelling wave solution of the modified Stefan problem with velocity $c^{*}$.

REMARK 2.1. It will be clear in the proof of Theorem 2.1 that the stretched phase field $\Phi^{\epsilon}(y) \equiv \varphi^{\epsilon}(\epsilon y)$ converges to $\Phi^{0}(y)$ uniformly on $\mathbb{R}$ as $\epsilon \downarrow 0$ (see Lemma 2.1 for the definition of $\Phi^{0}$ ). Here we define the stretched variable

$$
y=z / \epsilon .
$$

The precise meaning of the uniqueness result in Theorem 2.1 will also become clear in the proof by using this stretched coordinate. For small $\epsilon$, the equilibria of interest will be near

$$
L^{0}=\left(1,0, u_{\text {cool }}\right) \text { and } S^{0}=\left(-1,0, u_{\text {cool }}+l\right) .
$$

Integrating (2.4) from $z=-\infty$, one has

$$
u(z)=u_{s}^{\epsilon}-\frac{c}{K} \int_{-\infty}^{z} e^{-(c / K)(z-s)} \frac{l}{2}\left[\varphi(s)-\varphi_{-\infty}^{\epsilon}\right] d s .
$$

Substituting (2.17) into (2.2), one obtains

$$
\begin{aligned}
\epsilon^{2} \varphi_{z z} & +\alpha \epsilon^{2} c \varphi_{z}+g(\varphi) \\
& +\frac{2 \epsilon m}{\sigma_{0}}\left\{u_{s}^{\epsilon}+l-\frac{c}{K} \int_{-\infty}^{z} e^{-(c / K)(z-s)} \frac{l}{2}\left[\varphi(s)-\varphi_{-\infty}^{\epsilon}\right] d s\right\}=0
\end{aligned}
$$

which is subject to boundary conditions

$$
\varphi( \pm \infty)=\varphi_{ \pm \infty}^{\epsilon} \text { and } \varphi_{z}( \pm \infty)=0 .
$$

We note that the velocity $c$ is also an unknown variable in (2.18).

For subsequent discussions, it will be convenient to rewrite Eqs. (2.2), (2.4) using the stretched coordinate $y$ defined by $(2.15)$ and the "inner variables"

$$
\Phi(y) \equiv \varphi(\epsilon y) ; \quad U(y) \equiv u(\epsilon y) .
$$

Then, (2.18), (2.19) are rewritten in the equivalent form (for small $\epsilon$ ) as

$$
\begin{aligned}
\Phi_{y y} & +\alpha \epsilon c \Phi_{y}+g(\Phi) \\
& +\frac{2 \epsilon m}{\sigma_{0}}\left\{u_{s}^{\epsilon}+l-\frac{\epsilon c}{K} \int_{-\infty}^{y} e^{-(\epsilon c / K)(y-s)} \frac{l}{2}\left[\Phi(s)-\varphi_{-\infty}^{\epsilon}\right] d s\right\}=0,
\end{aligned}
$$




$$
\Phi( \pm \infty)=\varphi_{ \pm \infty}^{\epsilon}, \quad \Phi_{y}( \pm \infty)=0 .
$$

In view of (2.9) and (2.21), it is natural to take the solution to the following problem as a first approximation:

$$
\Phi_{y y}+g(\Phi)=0, \quad \Phi( \pm \infty)= \pm 1, \quad \Phi_{y}( \pm \infty)=0 .
$$

The following result is well known (see, for instance, [15]).

LemMa 2.1. There exists a unique solution $\Phi^{0}$ of $(2.23)$ with $\Phi^{0}(0)=0$ which is smooth, strictly increasing and satisfies the following

(a)

$$
\begin{aligned}
\left|\Phi^{0}(y) \pm 1\right| \leq C \exp (-\gamma|z|) & \text { as } z \rightarrow \mp \infty, \\
\left|\Phi_{y}^{0}(y)\right| \leq C \exp (-\gamma|z|) & \text { as } z \rightarrow \mp \infty,
\end{aligned}
$$

where $C$ and $\gamma$ are positive constants.

(b) With $\varphi^{0}(z) \equiv \Phi^{0}(z / \epsilon)$ one has

$$
\lim _{\epsilon \downarrow 0} \varphi^{0}(z)= \begin{cases}-1 & \text { uniformly on } z \in(-\infty, A], \\ 1 & \text { uniformly on } z \in(A, \infty),\end{cases}
$$

for any $A>0$.

We seek a solution of $(2.21),(2.22)$ in the form

$$
\Phi=\Phi^{0}+\Psi \text {. }
$$

Note that we do not put $\Phi=\Phi^{0}+\epsilon \Psi$, since such a setting will cause a difficulty in our framework of the solution space (see Remark 2.3). The appropriate function space for $\Psi$ will be specified in the next lemma. Substituting (2.24) into (2.21) one has

$$
\begin{gathered}
\Psi_{y y}+g^{\prime}\left(\Phi^{0}\right) \Psi+\alpha \epsilon c \Phi_{y}^{0}+\alpha \epsilon c \Psi_{y}+G\left(\Psi, \Phi^{0}\right) \\
+\frac{2 \epsilon m}{\sigma_{0}}\left\{u_{s}^{\epsilon}+l-\frac{\epsilon c}{K} \int_{-\infty}^{y} e^{-(\epsilon c / K)(y-s)} \frac{l}{2}\left[\Phi^{0}(s)+\Psi(s)-\varphi_{-\infty}^{\epsilon}\right] d s\right\}=0 \\
G\left(\Psi, \Phi^{0}\right) \equiv g\left(\Phi^{0}+\Psi\right)-g\left(\Phi^{0}\right)-g^{\prime}\left(\Phi^{0}\right) \Psi \\
\Psi( \pm \infty)=\epsilon \eta_{ \pm}(\epsilon), \quad \Psi_{y}( \pm \infty)=0
\end{gathered}
$$

We will solve $(2.25)$ by utilizing the alternative method. Let the space $\mathscr{B}^{k}(\mathbb{R})$ and the associated norm $\|f\|_{k}$ be defined by $\mathscr{B}^{k}(\mathbb{R})=\{f: \mathbb{R} \rightarrow \mathbb{R}$ has bounded and continuous derivatives up to the $k$ th order $\}$

$$
\|f\|_{k}=\sum_{j=0}^{k} \sup _{z \in \mathbb{R}}\left|D^{j} f(z)\right|
$$

We simply denote $\mathscr{B}^{0}(\mathbb{R}),\|\cdot\|_{0}$ by $\mathscr{B}(\mathbb{R}),\|\cdot\|$ and define $L: \mathscr{B}^{2}(\mathbb{R}) \rightarrow \mathscr{B}(\mathbb{R})$ by

$$
L \equiv \frac{d^{2}}{d y^{2}}+g^{\prime}\left(\Phi^{0}\right)
$$

so that $L$ is a continuous linear operator whose null space we denote by $\mathscr{N}(L)$. The following lemma is basic [10, p. 31], [11]. 
Lemma 2.2. The null space $\mathscr{N}(L)$ is one-dimensional and $\mathscr{N}(L)=\operatorname{span}\left\{\Phi_{y}^{0}\right\}$. For any $h \in \mathscr{B}(\mathbb{R})$, the equation

$$
L u+h=0
$$

has a solution in $\mathscr{B}(\mathbb{R})$ if and only if

$$
P h=0
$$

where $P$ is the projection onto $\mathscr{N}(L)$, i.e.,

$$
P h=\frac{1}{\left\|\Phi_{y}^{0}\right\|_{L^{2}(\mathbb{R})}} \Phi_{y}^{0} \int_{-\infty}^{\infty} \Phi_{y}^{0} h d y .
$$

Moreover, there is a bounded linear operator

$$
\mathscr{K}:(I-P) \mathscr{B}(\mathbb{R}) \rightarrow(I-P) \mathscr{B}^{2}(\mathbb{R})
$$

called the inverse of $L$ such that $L \mathscr{K}=I$ on $(I-P) \mathscr{B}(\mathbb{R})$ and $\mathscr{K} L=I-P$ on $\mathscr{B}^{2}(\mathbb{R})$.

Using Lemma 2.2 , one observes that $(2.25)$ is equivalent to the following system:

$$
\begin{gathered}
\Psi+\mathscr{K}(I-P) F(\Psi, c, \epsilon)=0, \\
P F(\Psi, c, \epsilon)=0,
\end{gathered}
$$

for $\Psi \in(I-P) \mathscr{B}^{2}(\mathbb{R})$ and

$$
\begin{aligned}
F(\Psi, c, \epsilon) \equiv & \alpha \epsilon c \Phi_{y}^{0}+\alpha \epsilon c \Psi_{y}+G\left(\Psi, \Phi^{0}\right) \\
& +\frac{2 \epsilon m}{\sigma_{0}}\left\{u_{s}^{\epsilon}+l-\frac{\epsilon c}{K} \int_{-\infty}^{y} e^{-(\epsilon c / K)(y-s)} \frac{l}{2}\left[\Phi^{0}(s)+\Psi(s)-\varphi_{-\infty}^{\epsilon}\right] d s\right\} .
\end{aligned}
$$

REMARK 2.2. In general the solution $\Psi$ of (2.25) has the decomposition $\Psi=$ $\eta+\chi, \eta \in \mathscr{N}(L), \chi \in(I-P) \mathscr{B}^{2}(\mathbb{R})$. However, we do not lose any generality in assuming that $\eta=0$ since the solution obtained with nonzero $\eta$ is simply a "translation," with respect to the $y$-direction, of that with $\eta=0$.

The next lemma is a consequence of direct computation.

Lemma 2.3. The mapping $H^{\epsilon}: \mathscr{B}(\mathbb{R}) \rightarrow \mathscr{B}^{1}(\mathbb{R})$ defined by

$$
H^{\epsilon}(\theta) \equiv \frac{\epsilon c}{K} \int_{-\infty}^{y} e^{(\epsilon c / K)(y-s)} \theta(s) d s
$$

is uniformly bounded with respect to $\epsilon$, so that

$$
\left\|H^{\epsilon}(\theta)\right\|_{1} \leq M\|\theta\|
$$

where $M$ is a positive constant independent of $\epsilon$. Moreover,

$$
\lim _{\epsilon \downarrow 0} \frac{d H^{\epsilon}}{d y}(\theta)=0 \quad \text { in } \mathscr{B}(\mathbb{R}) \text {-sense }
$$

holds for any given $\theta \in \mathscr{B}(\mathbb{R})$. 
REMARK 2.3. Note that for a given $\theta \in \mathscr{B}(\mathbb{R}), H^{\epsilon}(\theta)$ itself does not generally converge in $\mathscr{B}(\mathbb{R})$ as $\epsilon \downarrow 0$. In fact, when $\theta$ is a monotone increasing positive function satisfying

$$
\lim _{y \rightarrow-\infty} \theta(y)=0 \text { and } \lim _{y \rightarrow-\infty} \theta(y)=\theta^{*}>0
$$

then it is clear that

$$
\lim _{y \rightarrow-\infty} H^{\epsilon}(\theta)=0 \text { and } \lim _{y \rightarrow+\infty} H^{\epsilon}(\theta)=\theta^{*} .
$$

On the other hand, $H^{\epsilon}(\theta)$ converges to a constant function on any compact set on $\mathbb{R}$ as $\epsilon \downarrow 0$ since $d H^{\epsilon}(\theta) / d y \rightarrow 0$ in $\mathscr{B}(\mathbb{R})$. This implies that $H^{\epsilon}(\theta)$ has no limit in $\mathscr{B}(\mathbb{R})$ when $\epsilon \downarrow 0$.

Now we solve (2.28) for $\Psi$ as a function of $c$ and $\epsilon$.

Lemma 2.4. Let $I_{0}$ be an open bounded interval in $\mathbb{R}^{+}$. Then there is a positive constant $\epsilon_{0}$ such that (2.28) has a unique solution $\Psi=\Psi(c, \epsilon)$ in $\mathscr{B}^{2}(\mathbb{R})$ for

$$
(c, \epsilon) \in \wedge_{0} \equiv I_{0} \times\left[0, \epsilon_{0}\right]
$$

and $\Psi$ is a continuous function of $(c, \epsilon)$ in $\wedge_{0}$ and is continuously differentiable with respect to $c$.

Moreover, $\Psi$ (resp. $\partial \Psi / \partial c$ ) has the form $\Psi=\epsilon \widehat{\Psi}$ (resp. $\partial \Psi / \partial c=\epsilon \partial \widehat{\Psi} / \partial c$ ) with $\|\widehat{\Psi}\|_{2} \leq M$ (resp. $\|\partial \widehat{\Psi} / \partial c\|_{2} \leq M$ ) and hence satisfies

$$
\lim _{\epsilon \downarrow 0} \Psi(c, \epsilon)=0 \lim _{\epsilon \downarrow 0} \frac{\partial \Psi}{\partial c}(c, \epsilon)=0 \quad \text { in } \mathscr{B}^{2}(\mathbb{R}) \text {-sense. }
$$

Here, $M$ is a positive constant independent of $\epsilon$.

Proof. The first part is clear from the uniform contraction arguments and the smooth dependency of $F(\Psi, c, \epsilon)$ with respect to $c$. In fact, recalling (2.9), (2.10) we see that the principal part of $\Psi(c, \epsilon)$ for small $\epsilon$ is given by

$$
\begin{aligned}
\Psi(c, \epsilon) \simeq-\mathscr{K}(I-P)\left[\alpha \epsilon c \Phi_{y}^{0}\right. & \\
& \left.+\frac{2 \epsilon m}{\sigma_{0}}\left\{u_{\text {cool }}+l-\frac{c \epsilon}{K} \int_{-\infty}^{y} e^{-(\epsilon c / K)(y-s)} \frac{l}{2}\left(\Phi^{0}(s)+1\right) d s\right\}\right]
\end{aligned}
$$

and $\Psi(c, \epsilon)$ can be constructed by an iteration procedure starting from the above term. This also implies the bounds and limit for $\Psi$. Differentiating (2.28) with respect to $c$ and noting that from Lemma 2.3

$$
\frac{c \epsilon}{K} \int_{-\infty}^{y} \frac{\epsilon}{K}(y-s) e^{-(\epsilon c / K)(y-s)} \frac{l}{2}\left(\Phi^{0}(s)+1\right) d s
$$

also remains bounded in $\mathscr{B}(\mathbb{R})$ as $\epsilon \downarrow 0$, one obtains analogously the result for $\partial \Psi / \partial c$.

Substituting the expression $\Psi(c, \epsilon)$ from Lemma 2.4 into (2.29) we have the bifurcation equation with respect to $c$ and $\epsilon$,

$$
P F(\Psi(c, \epsilon), c, \epsilon)=0
$$


which we can write with $\langle$,$\rangle denoting the L^{2}$-inner product,

$$
\begin{aligned}
B(c, \epsilon) \equiv & \left\langle\alpha \epsilon c \Phi_{y}^{0}+\alpha \epsilon c \Psi_{y}(c, \epsilon)+G\left(\Psi(c, \epsilon), \Phi^{0}\right)\right. \\
& \left.+\frac{2 \epsilon m}{\sigma_{0}}\left\{u_{s}^{\epsilon}+l-\frac{\epsilon c}{K} \int_{-\infty}^{y} e^{(\epsilon c / K)(y-s)} \frac{l}{2}\left[\Phi^{0}(s)+\Psi(c, \epsilon)-\varphi_{-\infty}^{\epsilon}\right] d s\right\}, \Phi_{y}^{0}\right\rangle \\
= & 0 .
\end{aligned}
$$

Although it follows from Lemmas 2.3 and 2.4 that $B(c, \epsilon)$ and $\partial B / \partial c(c, \epsilon)$ are defined in $\wedge_{0}$, we cannot determine $c$ as a function of $\epsilon$ by using the implicit function theorem because of the fact that

$$
B(c, 0)=\frac{\partial B}{\partial c}(c, 0)=0 .
$$

However, by virtue of the exponentially decaying property of $\Phi_{y}^{0}$ (Lemma 2.1) we can show that

$$
\widetilde{B}(c, \epsilon) \equiv B(c, \epsilon) / \epsilon
$$

is well defined in $\wedge_{0}$ and the implicit function theorem is applicable to it as follows. Lemma 2.5. With $\wedge_{0}$ defined as in Lemma 2.4 , and $m$ defined by (1.7), $\widetilde{B}(c, \epsilon)$ and $\partial \widetilde{B} / \partial c(c, \epsilon)$ are well defined and continuous in $\wedge_{0}$. Moreover, one has

$$
\begin{gathered}
\widetilde{B}(c, 0)=\alpha c m+\frac{4 m}{\sigma_{0}}\left(u_{\text {cool }}+l\right), \\
\frac{\partial \widetilde{B}}{\partial c}(c, 0)=\alpha m>0 .
\end{gathered}
$$

Before proving this lemma we need the following result which deals with the integral term in (2.32).

Proposition 2.1. Let $\theta^{\epsilon}(y)$ be defined by

$$
\theta^{\epsilon}(y) \equiv \frac{\epsilon c}{K} \int_{-\infty}^{y} e^{-(\epsilon c / K)(y-s)} \frac{l}{2}\left[\Phi^{0}(s)+1\right] d s .
$$

Then $\theta^{\epsilon}(y)$ converges to zero uniformly on any compact set of $\mathbb{R}$ when $\epsilon \downarrow 0$.

Proof. Let $w^{\epsilon}(z)$ be a family of arbitrary functions which converge to $w^{0}(z)$ in $\mathscr{B}(\mathbb{R})$ with $w^{0}(0)=0$. Rewriting $w^{\epsilon}(z)$ with the stretched coordinate $y=z / \epsilon$, i.e., $W^{\epsilon}(y) \equiv w^{\epsilon}(\epsilon y)$, one sees that $W^{\epsilon}$ converges to zero uniformly on any compact set as $\epsilon \downarrow 0$. Thus, if we define $\vartheta^{\epsilon}(z)=\theta^{\epsilon}(z / \epsilon)$ and $\varphi^{0}(z)=\Phi^{0}(z / \epsilon)$ we see that $\vartheta^{\epsilon}$ and $\varphi^{0}$ satisfy

$$
\vartheta^{\epsilon}(z)=\frac{c}{K} \int_{-\infty}^{z} e^{-(c / K)(z-s)} \frac{l}{2}\left[\varphi^{0}(s)+1\right] d s .
$$

Using the uniform convergence on compact sets in conjunction with Lemma 2.1 one has

$$
\lim _{\epsilon \downarrow 0} \vartheta^{\epsilon}(z)=\vartheta^{0}(z)=\left\{\begin{array}{ll}
0, & \text { for } z \in(-\infty, 0], \\
l\left(1-e^{-(c / K) z}\right), & \text { for } z \in(0, \infty)
\end{array} \text { in } \mathscr{B}(\mathbb{R})\right. \text {-sense }
$$

which completes the proof of Proposition 2.1. 
Proof of Lemma 2.5. In view of (2.32), it is clear that the terms which must be examined are the integral term and $G\left(\psi(c, \epsilon), \Phi^{0}\right) / \epsilon$. Since $G$ is at least of quadratic order with respect to $\Psi$, it follows from Lemma 2.4 that $G\left(\Psi(c, \epsilon), \Phi^{0}\right) / \epsilon$ is well defined in $\wedge_{0}$ and satisfies

$$
\lim _{\epsilon \downarrow 0} G\left(\Psi(c, \epsilon), \Phi^{0}\right) / \epsilon=0 \quad \text { in } \mathscr{B}(\mathbb{R}) \text {-sense }
$$

and consequently, one has

$$
\lim _{\epsilon \downarrow 0}\left\langle G\left(\Psi(c, \epsilon), \Phi^{0}\right) / \epsilon, \Phi_{y}^{0}\right\rangle=0
$$

In a similar way one can show by Lemma 2.4 that

$$
\left\langle\frac{\partial}{\partial c} G\left(\Psi(c, \epsilon), \Phi^{0}\right) / \epsilon, \Phi_{y}^{0}\right\rangle=\left\langle G_{\Psi}\left(\Psi(c, \epsilon), \Phi^{0}\right) \frac{\partial \Psi}{\partial c}(c, \epsilon) / \epsilon, \Phi_{y}^{0}\right\rangle
$$

is well defined in $\wedge_{0}$ and tends to zero when $\epsilon \downarrow 0$.

The integral part, i.e.,

$$
\left\langle\frac{\epsilon c}{K} \int_{-\infty}^{y} e^{-(\epsilon c / K)(y-s)} \frac{l}{2}\left[\Phi^{0}(s)+\Psi(c, \epsilon)-\varphi_{-\infty}^{\epsilon}\right] d s, \Phi_{y}^{0}\right\rangle
$$

is clearly continuous for positive $\epsilon$ and $c$. In view of Remark 2.3 we cannot expect this term to converge a member of $\mathscr{B}(\mathbb{R})$. Nevertheless, Proposition 2.1 is sufficient for our purpose in examining the continuity at $\epsilon \downarrow 0$, since $\Phi_{y}^{0}$ decays exponentially as $|y| \rightarrow \infty$.

Utilizing Proposition 2.1, (2.10), and Lemmas 2.3 and 2.4, we see that (2.39) converges to zero as $\epsilon \downarrow 0$. Similarly, we can verify that the derivative of (2.39) with respect to $c$ is also well defined for $(c, \epsilon) \in \wedge_{0}$ and converges to zero as $\epsilon \downarrow 0$.

Thus, from (2.32) it is clear that

$$
\begin{aligned}
\lim _{\epsilon \downarrow 0} \widetilde{B}(c, \epsilon) & =\alpha c\left\langle\Phi_{y}^{0}, \Phi_{y}^{0}\right\rangle+\frac{2 m}{\sigma_{0}}\left(u_{\text {cool }}+l\right) \int_{-\infty}^{\infty} \Phi_{y}^{0} d y \\
& =\alpha c m+\frac{4 m}{\sigma_{0}}\left(u_{\text {cool }}+l\right) .
\end{aligned}
$$

This establishes (2.34). In a similar way, one can obtain (2.35), which completes the proof of Lemma 2.5 .

Proof of Theorem 2.1. We are now ready to solve $\widetilde{B}(c, \epsilon)=0$ by using the implicit function theorem. In view of $(2.34)$ it is natural to choose the limiting velocity, $c^{*}$, to be

$$
c^{*} \equiv \frac{-4}{\alpha \sigma_{0}}\left(u_{\text {cool }}+l\right)
$$

which is the velocity selected by the travelling wave in the modified Stefan problem [8], [9].

An application of the implicit function theorem to $\widetilde{B}(c, \epsilon)$ at $(c, \epsilon) \equiv\left(c^{*}, 0\right)$ implies a unique continuous solution $c(\epsilon)$ which satisfies $\widetilde{B}(c(\epsilon), \epsilon)$ and $c^{*}=$ $\lim _{\epsilon \downarrow 0} c(\epsilon)$. This implies the existence of unique solutions $\Phi^{\epsilon}(y)$ to $(2.21)$ and (2.22) in $\mathscr{B}^{2}(\mathbb{R})$ which converge to $\Phi^{0}(y)$ as $\epsilon \downarrow 0$. Returning to the original problem in the (unscaled) moving coordinate $z=x-c t$, recalling Lemma 2.1 and that 
$\varphi=\varphi^{0}+\widetilde{\Psi},\left[\widetilde{\Psi}(z) \equiv \Psi\left(\frac{z}{\epsilon}\right)\right]$ we obtain an $\epsilon$-family of travelling wave solutions of (2.18), (2.19) which satisfy

$$
\lim _{\epsilon \downarrow 0} \varphi^{\epsilon}= \begin{cases}-1 & \text { uniformly on } z \in(-\infty,-A] \\ 1 & \text { uniformly on } z \in(Z,+\infty),\end{cases}
$$

for any $A>0$, and

$$
\lim _{\epsilon \downarrow 0} u^{\epsilon}=\left\{\begin{array}{l}
u_{\text {cool }}+l \text { on } z \leq 0, \\
u_{\text {cool }}+l e^{\left(c^{*} / K\right)\left(x-c^{*} t\right)} \quad \text { on } z>0,
\end{array}\right.
$$

uniformly on $\mathbb{R}$ with respect to the measure induced by the travelling coordinate $z$.

It follows from (2.40) and (2.42) that $u^{\epsilon}$ converges to the planar travelling wave solution of the modified Stefan problem (surface tension and kinetics) (1.9), (1.10), (1.12) with $v=c^{*}$ and $\Delta s \equiv 4$ and $\sigma_{0} \equiv \xi a^{-1 / 2} m$ is the surface tension parameter (1.7).

3. Physical interpretation and nonexistence of travelling wave solutions in the classical Stefan limit. We can interpret the bifurcation equation in a physical context. For this purpose, it is convenient to rewrite $B(c, \epsilon)$ (not $\widetilde{B}(c, \epsilon)$ ) using the original moving coordinate $z$, so that

$$
\begin{aligned}
b(c, \epsilon) \equiv & \left\langle\alpha \epsilon c \varphi_{z}^{0}+\alpha \epsilon c \psi_{z}(c, \epsilon)+\widehat{G}\left(\psi(c, \epsilon) ; \varphi^{0}\right)\right. \\
& \left.+\frac{2 m}{\sigma_{0}}\left\{u_{s}^{\epsilon}+l-\frac{c}{K} \int_{-\infty}^{z} e^{-(c / K)(z-s)} \frac{l}{2}\left[\varphi^{0}(s)+\psi(c, \epsilon)-\varphi_{-\infty}^{\epsilon}\right] d s\right\}, \varphi_{z}^{0}\right\rangle \\
= & 0
\end{aligned}
$$

where $\psi$ and $\widehat{G}$ correspond to $\Psi$ and $\frac{G}{\epsilon}$ in the $z$-coordinates. In view of Lemma 2.4 and the identity $\epsilon \partial / \partial z=\partial / \partial y$, we see that $\epsilon \psi_{z}(c, \epsilon)$ and $\widehat{G}$ as well as their $c$-derivatives tend to zero in the supremum norm when $\epsilon \downarrow 0$. On the other hand, it should be noted that $\varphi_{z}^{0}$ does not remain an ordinary function as $\epsilon \downarrow 0$, but converges to a "Dirac point mass." In fact,, $\varphi_{z}^{0}$ has a sharp peak at $z=0$ and its total mass is constant, i.e., $\int_{-\infty}^{\infty} \varphi_{z}^{0}(z) d z=2$ is independent of $\epsilon$. More precisely, one has the following results proved in Lemma 2.3 of [12].

Proposition 3.1. The function $\varphi^{0}$ in Lemma 2.1 satisfies

$$
\lim _{\epsilon \downarrow 0} \varphi_{z}^{0}=2 \delta_{0} \quad \text { in } \mathscr{B}^{\prime}(\mathbb{R}) \text {-sense }
$$

where $\delta_{0}$ is the Dirac point mass at $z=0$, i.e., one has

$$
\lim _{\epsilon \downarrow 0}\left\langle\varphi_{z}^{0}, h\right\rangle=2 h(0) \quad \text { for } h \in \mathscr{B}(\mathbb{R}) .
$$

Using Proposition 3.1, Lemma 2.1(b) and noting that

$$
\left\|\Phi_{y}^{0}\right\|_{L^{2}(\mathbb{R})}^{2}=\int_{-\infty}^{\infty} \epsilon\left(\varphi_{z}^{0}(z)\right)^{2} d z
$$


we reach the same results as in Lemma 2.5 for $b(c, \epsilon)$, and hence Theorem 2.1. In particular, recalling that the temperature distribution is given by

$$
u^{\epsilon}(z)=u_{s}^{\epsilon}+l-\frac{c}{K} \int_{-\infty}^{z} e^{-(c / K)(z-s)} \frac{l}{2}\left[\varphi^{0}(s)+\psi(c(\epsilon), \epsilon)-\varphi_{-\infty}^{\epsilon}\right] d s
$$

it is apparent from (2.9) and (2.38) that the inner product

$$
\left\langle\left\{u_{s}^{\epsilon}-\frac{c}{K} \int_{-\infty}^{z} e^{-(c / K)(z-s)} \frac{l}{2}\left[\varphi^{0}(s)+\psi(c(\epsilon), \epsilon)-\varphi_{-\infty}^{\epsilon}\right] d s\right\}, \varphi_{z}^{0}\right\rangle
$$

converges to the temperature at the interface $u_{\text {cool }}+l$. The bifurcation equation $b(c, \epsilon)=0$ then implies the temperature-velocity relation $(2.40)$.

Thus the temperature across the interface satisfies this relation in an "averaged" sense with respect to a measure induced by $\varphi^{0}$.

Next, we consider the other distinguished limits mentioned in Sect. 1 which give rise to different sharp interface models, e.g., the classical Stefan (CS) and the alternative modified Stefan (AMS) models. In the pure one-dimensional case these two sharp interface models are identical although the stability properties of any planar solution with respect to higher-dimensional space can be expected to vary between the two models.

It is easy to verify [8], [9] that the CS and AMS models do not have planar travelling (i.e., constant velocity) solidification waves, subject to (2.11) and hence one expects the same situation in each distinguished limit of the phase field equations. In fact one can prove the following nonexistence result.

THEOREM 3.1. In the distinguished limit of the classical Stefan (1.9)-(1.11) or the alternative modified Stefan ((1.9), (1.10), (1.12) with $\alpha=0)$ limits, the phase field model (1.2), (1.8) does not have an $\epsilon$-family of travelling waves $\left(\varphi^{\epsilon}, u^{\epsilon}, c(\epsilon)\right)$ which satisfies (2.11) and remains bounded in terms of the stretched coordinate $y=\frac{z}{\epsilon}$ in $\mathscr{B}^{2}(\mathbb{R}) \times \mathscr{B}(\mathbb{R}) \times \mathbb{R}^{+}$for $\epsilon \in\left(0, \epsilon_{0}\right)$.

Proof. We prove this theorem by contradiction. First, in view of (2.21), Lemma 2.1 and that $\epsilon m / \sigma_{0} \quad(=\epsilon / \hat{\sigma}=a)$ tends to zero, we can assume without loss of generality that $\Phi^{\epsilon}(y)\left(\equiv \varphi^{\epsilon}(\epsilon y)\right)$ converges to $\Phi^{0}$ as $\epsilon \downarrow 0$. For if it does not, we can extract a convergent subsequence from $\left\{\Phi^{\epsilon}\right\}$ by using the Ascoli-Arzela theorem. Hence, we can write $\Phi^{\epsilon}$ as

$$
\Phi^{\epsilon}=\Phi^{0}+\Psi^{\epsilon}
$$

where $\Psi^{\epsilon}$ approaches zero in the $\mathscr{B}^{2}(\mathbb{R})$-sense as $\epsilon \downarrow 0$. Now all but one of the procedures and previous lemmas used in obtaining the bifurcation equation (3.32) are also valid for these two distinguished limits. In particular, one needs a modification of Lemma 2.4 consisting of replacing

$$
\Psi=\epsilon \widehat{\Psi},\|\widehat{\Psi}\|_{2}<M \quad \text { by } \Psi=\frac{\epsilon}{\hat{\sigma}} \widehat{\Psi}, \quad\|\widehat{\Psi}\|_{2}<M .
$$

Recall again that $\epsilon / \hat{\sigma} \quad(=a)$ tends to zero. However, the resulting bifurcation equation (2.32) is not valid for small $\epsilon$. In fact, dividing both sides of (2.32) for the modified Stefan case by $\epsilon / \hat{\sigma}$ we have

$$
\left\langle\alpha \hat{\sigma} c \Phi_{y}^{0}+\alpha \hat{\sigma} c \Psi_{y}^{\epsilon}+\widetilde{G}+2\left\{u_{s}^{\epsilon}+\cdots\right\}, \Phi_{y}^{0}\right\rangle=0
$$


where $\widetilde{G} \equiv G /(\epsilon / \hat{\sigma})$. It is clear that $(3.3)$ does not hold for small $\hat{\sigma}$, since the lefthand side of $(3.3)$ converges to $4\left(u_{\text {cool }}+l\right) \neq 0$ [by (2.11)] when $\hat{\sigma} \downarrow 0$. A similar contradiction occurs when the scaling limit of AMS is considered. This completes the proof of Theorem 3.1 .

\section{REFERENCES}

[1] G. Caginalp, Mathematical models of phase boundaries, Material Instabilities in Continuum Problems and Related Mathematical Problems (Ed. J. Ball), Heriot-Watt Symposium 1985-1986, Oxford Publ. (1988)

[2] G. Caginalp, Stefan and Hele-Shaw type models as asymptotic limits of the phase field equations, Phys. Rev. A 39, 5887-5896 (1989)

[3] L. I. Rubinstein, The Stefan problem, Transl. Math. Monographs, Vol. 27, American Mathematical Society, Providence, Rhode Island, 1971

[4] G. Caginalp and P. C. Fife, Elliptic problems involving phase boundaries satisfying a curvature condition, IMA J. Appl. Math. 38, 195-217 (1987)

[5] R. Kohn and P. Sternberg, Local minimizers and singular perturbations, Proc. Roy. Soc. Edinburgh, Sect. A 111, 69-84 (1989)

[6] N. Alikakos and P. Bates, On the singular limit in a phase field model of a phase transition, Ann. Inst. H. Poincaré Non Linéaire 5, 141-178 (1988)

[7] S. Luckhaus and L. Modica, The Gibbs-Thompson relation within the gradient theory of phase transitions, Arch. Rational Mech. Anal. 107, 71-83 (1989)

[8] J. N. Dewynne, S. D. Howison, J. R. Ockendon, and W. Xie, Asymptotic behavior of solutions to the Stefan problem with a kinetic condition at the free boundary, J. Austral. Math. Soc. Ser. B 31, 81-96 (1989)

[9] G. Caginalp and J. Chadam, Stability of interfaces with velocity correction term, to appear in Rocky Mountain J. Math.

[10] S.-N. Chow and J. Hale, Methods of Bifurcation Theory, Springer, Berlin, 1982

[11] S.-N. Chow, J. Hale, and J. Mallet-Paret, An example of bifurcation to homeoclinic orbits, J. Differential Equations 37, 351-373 (1980)

[12] Y. Nishiura and H. Fujii, Stability of singularly perturbed solutions to systems of reaction-diffusion equations, SIAM J. Math. Anal. 18, 1726-1770 (1987)

[13] J. W. Wilder, Travelling wave solutions for interfaces arising from phase boundaries based on a phase field model, Rensselaer Polytechnic Inst. preprint

[14] H. Fujii, Y. Nishiura, M. Mimura, and R. Kobayashi, Existence of curved fronts for the phase field model, In preparation

[15] P. C. Fife and J. B. McLeod, The approach of solutions of nonlinear diffusion equations to travelling front solutions, Arch. Rational Mech. Anal. 65, 335-361 (1977) 